\title{
Seleção de assistentes para a disciplina de programação OO: um estudo de caso sobre os dados da experiência anterior do monitor
}

\author{
Samara S. S. Sampaio ${ }^{1}$, Lívia M. R. Sampaio ${ }^{1}$, Matheus Gaudencio ${ }^{1}$ \\ ${ }^{1}$ Universidade Federal de Campina Grande (UFCG) \\ Caixa Postal 10.106 58429-900 - Campina Grande, PB - Brasil \\ samara.sampaio@ccc.ufcg.edu.br \\ \{livia, matheusgr\}@computacao.ufcg.edu.br
}

\begin{abstract}
Students who work as teaching assistants perform an essential pedagogical role in programming courses. These assistants create summative and formative feedback for students and are selected based upon different factors, such as previous experiences in the course and the subject. In this work, we evaluate the data about teaching assistance in a case study that focus on previous experiences and results of the feedback given by these assistants. We found that the different previous experiences of students did not affect the summative feedback and had little impact on the length of the formative feedback.
\end{abstract}

Resumo. Os alunos assistentes em cursos, especialmente de programação, realizam uma função importante de apoio pedagógico. Estes monitores atuam especialmente na criação de feedback somativo e formativo para os estudantes e são selecionados por diferentes aspectos, como experiências prévias no curso e na disciplina. Neste trabalho avaliamos, em um estudo de caso, os dados sobre a monitoria: experiências prévias e resultados dos feedbacks dado por esses assistentes. Encontramos que, as diferentes experiências prévias dos alunos não afetaram o feedback somativo que é gerado, e impactaram pouco o feedback formativo dos alunos que já realizaram assistência anteriormente.

\section{Introdução}

Aprender a programar requer atenção já que muitos estudantes apresentam dificuldades para entender conceitos mais abstratos, como por exemplo, Programação Orientada a Objeto (POO). As linguagens que utilizam POO requerem uma nova maneira de pensar e maior profundidade de conhecimento, podendo assim tornar mais complexo o processo de aprendizagem [Mirza et al. 2019].

As práticas de ensino e de aprendizagem requerem cuidados especiais, uma vez que as dificuldades apresentadas são os motivos de elevadas taxas de insucesso ou desistência dos alunos [Gomes 2010]. Diante disso, existe a necessidade do ensino prático com um acompanhamento contínuo dos resultados das atividades produzidas, como através de feedback que informe aos alunos se suas atividades estão sendo produzidas corretamente e como melhorar. A forma como o feedback é elaborado é essencial para sua eficácia no processo de aprendizagem do aluno [Hattie and Timperley 2007].

Nesse contexto, uma estratégia muito comum para apoiar o processo de ensinoaprendizagem na graduação é a inclusão de graduandos como assistentes de ensino (alunos assistentes). Alguns estudos demonstram que as universidades com programas de 
alunos assistentes, voltados para disciplinas de introdução a programação, apresentam resultados acadêmicos positivos [Mirza et al. 2019, Figuerêdo et al. 2021, Silva 2021]. Esses alunos auxiliam em diversas práticas como geração de material didático, esclarecimento de dúvidas sobre o conteúdo da disciplina tanto em sala de aula ou extra-classe, acompanhamento das atividades relacionadas à disciplina e feedback [Mirza et al. 2019]. O feedback pode ser somativo, de caráter mais quantitativo e representado por uma nota resultante da avaliação do aprendizado do aluno; e também formativo, de caráter mais qualitativo, que orienta o aluno sobre o seu processo de aprendizagem indicando pontos a serem melhorados [Santos and Pinto 2018].

Comumente, o perfil de aluno assistente buscado é aquele que obteve um bom desempenho quando cursou a disciplina alvo, que já teve experiências anteriores como aluno assistente ou que esteja mais adiantado no curso. Além disso, deseja-se qualidades como: saber se expressar, ter boa didática, saber ouvir, ter empatia pelos alunos com mais dificuldade e ser prestativo [Mirza et al. 2019, Figuerêdo et al. 2021]. Alguns métodos de recrutamento são mais, ou menos, restritivos quanto a tais características ou mesmo demonstram preocupação com questões de diversidade [Mirza et al. 2019]. Porém, pouco se sabe, de fato, como tais características influenciam no feedback fornecido pelos alunos assistentes.

Este trabalho teve o objetivo de estudar a influência de diferentes características dos assistentes no feedback fornecido por eles no contexto de uma disciplina de POO em um curso superior no Brasil. Trata-se de um estudo descritivo para identificar possíveis diferenças entre o feedback fornecido pelos assistentes e a relação disto com suas especificidades. No caso, foram consideradas características relacionadas com as experiências acadêmicas do aluno assistente. Ao todo, os dados incluíram 28 assistentes e em torno de 100 alunos matriculados na disciplina. Os resultados mostraram que não existe diferença entre a avaliação somativa feita pelos diferentes assistentes.

Por outro lado, quando consideramos o feedback formativo, avaliou-se o tamanho do feedback gerado e houveram diferenças considerando a experiência anterior como assistente, mas, estatisticamente não significativa. Destes resultados, não parece mandatório ser mais restritivo em relação às características do aluno assistente quando observamos o feedback que ele pode produzir. Outros fatores podem ser mais relevantes como qualidades ou mesmo o processo de treinamento e acompanhamento desses alunos, bem como é preciso ainda investigar sobre a qualidade do feedback formativo gerado.

Este trabalho está estruturado da seguinte forma. Na Seção 2 discutiremos os trabalhos relacionados, na Seção 3 a metodologia que foi utilizada, na Seção 4 os resultados e discussões, e por fim, as conclusões.

\section{Trabalhos relacionados}

O uso de graduandos como alunos assistentes em programas de monitoria (ou assistência de ensino) é uma prática comum nas instituições de ensino no Brasil e no mundo, com resultados positivos para o assistente, para o aluno assistido, para o professor e para a instituição [Mirza et al. 2019, Figuerêdo et al. 2021, Silva 2021].

No contexto do ensino de programação, os alunos costumam realizar várias atividades práticas para ter um melhor aprendizado e, os alunos assistentes desempenham 
um papel importante no acompanhamento dessas atividades provendo feedback que ajuda os alunos assistidos a saberem o que estão errando e como melhorarem. De acordo com Hattie e Timperley [Hattie and Timperley 2007] o feedback pode cumprir esse propósito instrucional, mas para isso precisa fornecer informações especificamentes relacionadas à atividade ou ao processo de aprendizagem que preenche uma lacuna entre o que é compreendido e o que se pretende compreender.

O processo de seleção ou recrutamento dos alunos assistentes pode variar muito dependendo da instituição de ensino, sendo mais, ou menos, restritivos, alguns com maior ênfase em diversidade ou prezando por inclusão. Mirza et al. [Mirza et al. 2019] analisa práticas sobre o tema recrutamento discutidas na literatura no contexto de UTAs (Undergraduate Teaching Assistants). Dentre tais práticas foram citadas o desempenho dos alunos na disciplina alvo, mecanismos para atrair bons alunos, como proceder nas entrevistas, mecanismos para melhorar diversidade e uma série de qualidades desejadas para o aluno. Por exemplo, o trabalho de Figuerêdo et al. [Figuerêdo et al. 2021] cita o processo de seleção descrito que inclui uma fase inicial onde é considerado o desempenho do candidato na disciplina alvo e uma carta de intenções. Após essa etapa, alguns candidatos são submetidos a uma entrevista onde devem preparar uma apresentação sobre determinado tópico e responder sobre o mesmo. Por fim, a última etapa consiste em avaliar as habilidades do candidato quanto ao feedback a ser dado ao aluno simulando a avaliação de uma atividade da disciplina.

No Brasil, a literatura sobre a monitoria ou assistência de ensino é mais focada nas experiência do monitor e do aluno nesse contexto [Figuerêdo et al. 2021, Silva 2021], não há muita atenção ao processo de recrutamento. De forma geral, o processo de seleção é focado no desempenho do aluno sobre o conteúdo da disciplina alvo, tanto quando cursou a mesma ou por meio de uma prova realizada no momento da seleção.

Seja qual for o processo de seleção de alunos assistentes, buscam-se aqueles com melhores pontuações no processo. Intuitivamente, acredita-se que, dessa forma, é possível obter os melhores resultados com o programa de assistência de ensino. Neste trabalho nós olhamos para o processo de recrutamento de alunos assistentes sobre uma nova perspectiva, analisando como e se as diferentes experiências dos alunos influenciam no feedback produzido por esses alunos.

\section{Metodologia}

O estudo segue uma abordagem quantitativa, tendo o objetivo de investigar relações entre características associadas com as experiências acadêmicas dos alunos assistentes, além do gênero, e feedbacks gerados pelos mesmos.

\subsection{Contexto}

Considerando o curso de Bacharelado em Ciência da Computação da UFCG, em 2019, aproximadamente $50 \%$ das disciplinas ofertadas para a graduação possuíam alunos assistentes. Ao todo foram cerca de 140 assistentes por semestre letivo, provenientes do programa institucional de monitoria, como também, de disciplinas de práticas de ensino em Ciência da Computação presentes na grade curricular do curso. Nessa instituição, o programa de monitoria seleciona a partir do rendimento acadêmico do aluno no curso e na disciplina específica, além de nota em uma prova de conteúdo específico. Já o processo de 
seleção para a disciplina de práticas de ensino busca aumentar a diversidade de monitores e na convocação de alunos mais experientes, mesmo que tenham tido menor desempenho na própria disciplina em que atuarão. As disciplinas iniciais de programação reúnem a maior parte desses assistentes, a exemplo de Laboratório de Programação II (LP2).

A disciplina LP2 ensina, na prática, os conceitos de POO aprendidos na disciplina de Programação II (P2), que é especialmente focada nos conhecimentos teóricos. Em LP2 são desenvolvidas atividades práticas individuais que são conhecidas por laboratórios, um projeto em grupo e provas. Os assistentes auxiliam os alunos no desenvolvimento das atividades tanto durante as aulas quanto em horários extra-classe.

Os assistentes também são responsáveis por fornecer feedback das atividades. Esse feedback é composto por duas partes: somativo e formativo. A primeira é feita de maneira guiada e gerando uma nota que varia entre 0 e 10 . Para isto, os professores disponibilizam uma planilha com orientações sobre os critérios a serem observados ao analisarem o código dos alunos e uma pontuação associada a cada critério, caso seja atendido corretamente ou quando apresentar algum tipo de erro, também identificados na planilha. Já na segunda parte, do feedback formativo, o assistente fica livre para escrever um comentário sobre o desempenho do aluno na atividade e dicas de como melhorá-la.

\subsection{Questões de estudo}

Para atender aos objetivos elaborados para essa pesquisa, buscamos responder se há diferença e/ou correlação entre a experiência prévia do aluno assistente e a quantidade ou valor do feedback formativo e somativo gerado.

\subsection{Dados}

Utilizamos dados do período 2019.2 da disciplina LP2. As amostras colhidas foram provenientes de 3 atividades de Laboratório (Lab 2, Lab 3 e Lab 4), tendo sido realizadas por, respectivamente, 119, 119 e 117 alunos. A primeira atividade da disciplina não é considerada pois não é avaliada pelos assistentes. Havia 28 assistentes atuando na disciplina nesse semestre. Foram realizadas análises dos feedbacks de cada atividade, isoladamente, como também dos dados acumulados das 3 atividades, a qual intitulamos de Labs.

Para realizar as análises com as informações obtidas nos feedbacks, utilizamos os seguintes dados dos alunos assistentes:

- Vínculo (Dado categórico)

- Monitoria Institucional: Os interessados se inscrevem em um processo seletivo e realizam uma avaliação baseada em prova, desempenho na disciplina e desempenho acadêmico, sendo aprovados os que obtiverem um melhor resultado e de acordo com a quantidade de vagas disponíveis;

- Prática: Matriculado nas disciplinas de Prática de Ensino os interessados se matriculam sem a necessidade de processo seletivo formal, apenas consulta ao professor da disciplina e que busca incentivar a presença de alunos de experiências mais diversas.

- Tipo de experiência anterior como aluno assistente (EAA - Dado categórico)

- Institucional: Alunos que já possuíram vínculo de monitoria;

- Prática: Alunos que já cursaram a disciplina de prática de ensino; 
- Institucional + Prática: Alunos que possuem os dois tipos de experiências citadas acima;

- Nenhuma: Alunos sem experiência prévia de assistência na disciplina.

- Experiência no curso (EC - Dado categórico)

- Nível 1: Aprovados nas disciplinas P2 e LP2;

- Nível 2: Aprovados também nas disciplinas de Estrutura de Dados e Laboratório de Estrutura de Dados (disciplinas do $3^{\circ}$ período);

- Nível 3: Aprovados em todas as anteriores e na disciplina de Projeto de Software (disciplina do $4^{\circ}$ período).

- Notas de aprovação nas disciplinas de P2 e LP2 (Dado numérico)

\subsection{Análise dos dados}

O intuito do estudo é investigar se o aluno assistente que possui experiências anteriores desempenha as atividades de forma diferente dos inexperientes. Para isso, realizamos primeiramente uma análise exploratória em cada experiência/característica analisada, para organizar e entender os dados, não descartando nenhuma informação nesse processo. Nessa etapa construímos gráficos para observarmos o comportamento dos valores de médias e medianas, buscando verificar se é evidente algum comportamento característico.

Posteriormente, para os dados categóricos, realizamos dois tipos de testes não paramétricos para verificar se as amostras se originam da mesma distribuição, ou seja, se as amostras sugerem diferenças entre as populações, indicando se os feedback possuem agrupamentos distintos de acordo com as experiências e características dos assistentes. Utilizamos o teste de Kruskal-Wallis quando observamos 3 ou mais grupos e o teste Mann Whitney para 2 grupos. Caso existisse diferença entre as amostras, realizamos o teste post-hoc de Dunn, para identificar qual era o grupo diferente.

Para verificar se existe alguma relação entre as notas de P2 e LP2 no feedback somativo ou no tamanho do feedback formativo utilizamos correlação de Pearson, visto que, nessa situação as variáveis dependente e independente são numéricas.

\section{Resultados}

\subsection{Vínculo}

Dos alunos assistentes 11 estavam vinculados como Prática/ Didática e 17 como monitoria institucional. As Figuras 1 e 2 mostram a distribuição do feedback somativo e o tamanho do feedback formativo, respectivamente.

O feedback somativo atribuído pelos assistentes vinculados como Monitoria Institucional possui maior dispersão no Lab 4, bem como é a atividade que apresenta uma maior distinção em relação à mediana. $\mathrm{O}$ feedback formativo apresenta uma maior quantidade de outliers em relação ao somativo. No entanto, não apresenta uma visível diferença na dispersão ou nas medianas das atividades. Para investigar se há alguma diferença significativa, realizamos o teste de Mann-Whitney porque a variável categórica possui apenas dois tipos: monitoria e prática. As hipóteses definidas foram:

- H0: Não há diferença significativa entre os tipos de vínculo;

- H1: Existe diferença significativa entre os tipos de vínculo. 


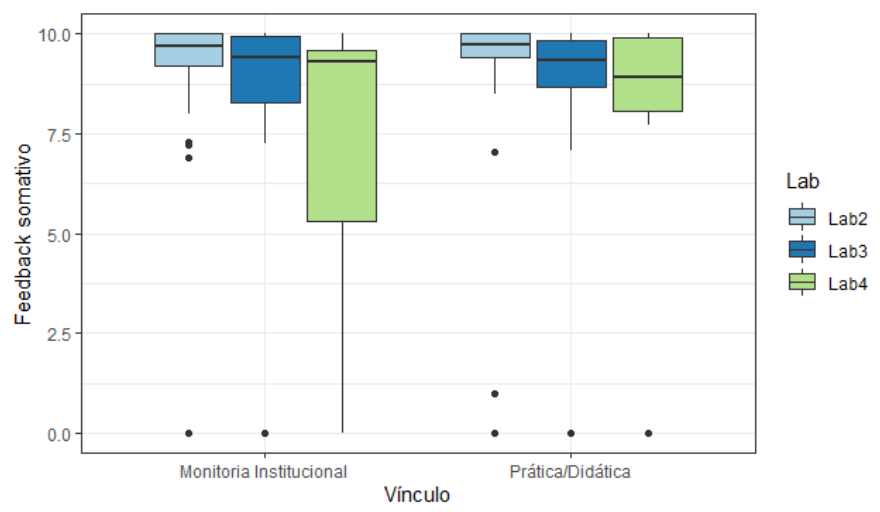

Figura 1. Feedback somativo de acordo com o vínculo do assistente.

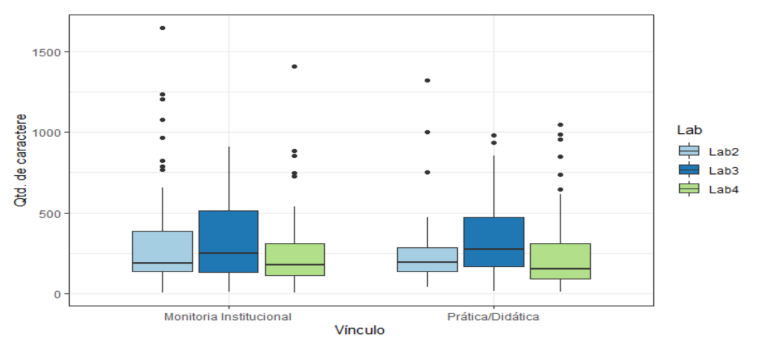

Figura 2. Tamanho do feedback formativo de acordo com o vínculo do assistente.

H0 é rejeitado caso p-value seja menor que 0,05 . Tanto nas três atividades de laboratório analisadas individualmente como quando olhamos todas as respostas juntas (labs) o p-value foi superior a 0,05 no feedback somativo e no tamanho do feedback formativo. Então a hipótese rejeitada é $\mathrm{H} 1$ e concluímos que não há diferença entre o feedback somativo ou tamanho do feedback formativo atribuído pelos diferentes tipos de vínculo dos alunos assistentes.

\subsection{Tipo de experiência anterior como aluno assistente - EEA}

Dos 28 alunos assistentes, 14 possuiam experiência anterior com a monitoria institucional, 2 com a disciplina de práticas de ensino, 3 institucional e prática, e 9 não possuiam nenhum tipo de experiência. As Figuras 3 e 4 mostram como se comportam o feedback somativo e o tamanho do feedback formativo de acordo com esse tipo de experiência.

As notas atribuídas no feedback somativo não apresentaram variações significativas, é possível perceber que a dispersão é mais distinta nos assistentes que possuem experiência institucional e prática da disciplina. No entanto, as medianas são bem próximas nos diferentes tipos de experiência anterior. Em relação ao tamanho do feedback formativo, observamos maior dispersão da quantidade de caracteres e maior diferença da mediana.

Para saber se essa diferença é significativa realizamos o teste inferencial de diferença Kruskal Wallis porque a variável categórica, EAA, possui mais de dois agrupamentos.As hipóteses definidas foram:

- H0: Não há diferença entre os feedbacks somativos ou o tamanho dos feedbacks 


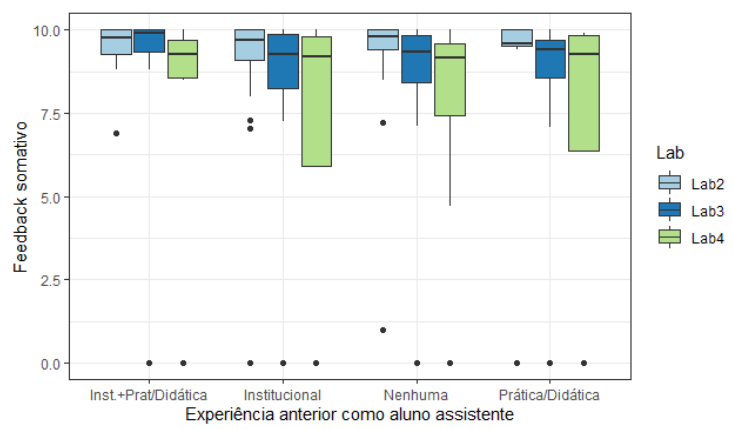

Figura 3. Feedback somativo de acordo com a EAA.

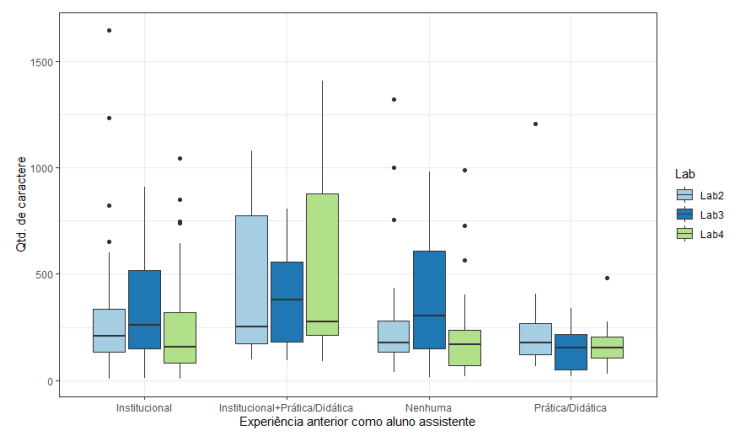

Figura 4. Tamanho do feedback formativo de acordo com a EAA.

formativos atribuídos pelos diferentes tipos de experiência anterior do aluno assistente;

- H1: Há pelo menos um tipo de experiência anterior do aluno assistente com feedbacks somativos ou tamanho dos feedbacks formativos diferentes

H0 é rejeitado caso $p$-value seja menor que 0,05 . Para o feedback somativo, os valores obtidos nas atividades Lab 2, Lab 3, Lab 4 e Labs foram: 0, 98, 0, 25, 0, 82 e 0, 22, respectivamente. Então a hipótese rejeitada é H1 e concluímos que não há diferença entre os feedbacks somativos atribuídos pelos diferentes tipos de experiência anterior dos alunos assistentes.

Para o tamanho do feedback somativo os p-values obtidos foram: 0, 10 no Lab 2, 0, 15 no Lab 3, 0, 07 no Lab 4 e 0, 005 no Labs. Apenas quando analisamos os dados acumulados (Labs), o p-value foi inferior a 0,05 , indicando que há pelo menos um tipo de experiência anterior do aluno assistente com tamanho do feedback formativo diferente. Para investigar melhor qual tipo de experiência possui uma diferença, foi realizado um teste post-hoc, teste de Dunn, o qual indicou que há diferença nos assistentes que já possuem experiência anterior Institucional + Prática. A figura 5 mostra que o $p$-value entre esse tipo de experiência e com os demais foi inferior a 0,05 .

\subsection{Experiência no curso - EC}

Quanto a experiência no curso, 6 assistentes estavam no Nível 1, 13 no Nível 2 e 9 no Nível 3. A Figura 6 mostra que a maior diferença se encontra na amplitude do feedback atribuído no Lab4 pelos assistentes que estão no Nível 2, isso pode ter ocorrido pela 


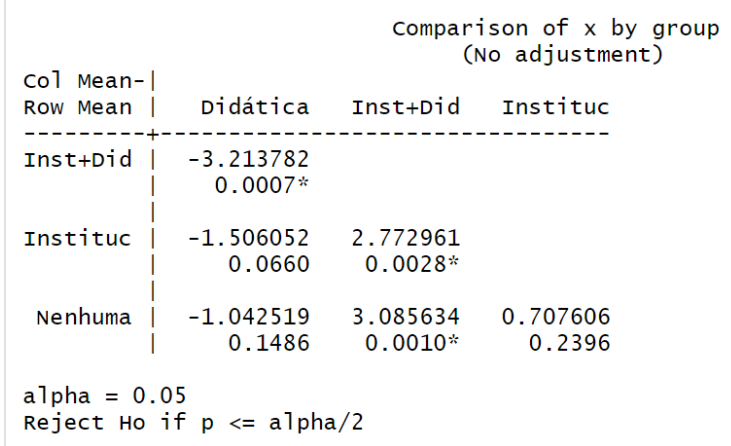

Figura 5. Teste de Dunn entre o feedback somativa e a EAA.

maior quantidade de alunos assistente com essa experiência Esse grupo também ficou responsável por uma grande quantidade de alunos que não entregaram o laboratório, logo, puxando o boxplot para a nota zero. Já na Figura 7 a dispersão do tamanho do feedback formativo não varia significativamente na amplitude, existindo mais outliers e pequena variação na amplitude e medianas.

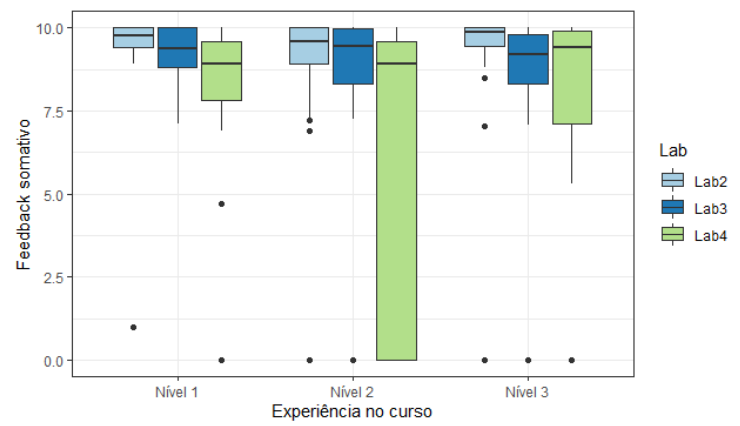

Figura 6. Feedback somativo de acordo com a EC.

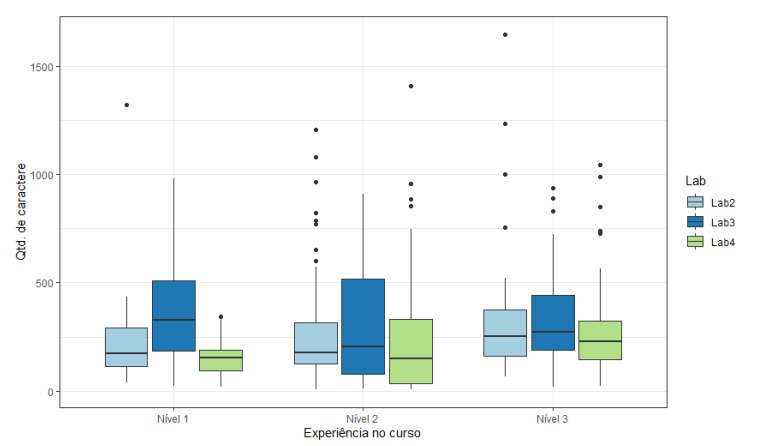

Figura 7. Tamanho do feedback formativo de acordo com a EC.

Realizamos os testes inferenciais de diferença utilizando Kruskal Wallis porque a variável categórica possui mais de dois agrupamentos (Níveis 1, 2 e 3). As hipóteses são:

- H0: Não há diferença entre o feedback somativo atribuído pelos diferentes tipos de experiência no curso; 
- H1: Há pelo menos um tipo de experiência no curso do aluno assistente com feedback somativo diferente.

Rejeitamos H0 caso $p$-value seja menor que 0,05 . Analisando o feedback somativo, tanto nas três atividades de laboratórios analisadas individualmente como quando olhamos todas as respostas junto (Labs) o p-value foi superior a 0,05. Então a hipótese rejeitada é $\mathrm{H} 1$ e concluímos que não há diferença entre o feedback somativo atribuído pelos diferentes tipos de experiência no curso dos alunos assistentes. No feedback formativo, apenas quando quando analisamos os dados acumulado foi possível identificar que existe diferença e com o teste de Dunn observamos que os assistentes mais experientes (nível 3) tendem a dar um feedback de maior tamanho.

\subsection{Desempenho nas disciplinas P2 e LP2}

A Figura 8 mostra a relação entre o feedback somativo e nota do assistente em P2, observamos que o feedback somativo não apresenta um crescimento ou decrescimento de acordo com a variação na nota da disciplina. Isso significa que não existe uma correlação aparente entre as variáveis. O coeficiente de correlação de Pearson, obtido nas três atividades analisadas individualmente e com os dados acumulados, foi bem próximo de 0 , confirmando que a correlação é inexistente. O mesmo ocorre com o tamanho do feedback formativo, como mostra a Figura 9. Para as notas na disciplina de LP2, ocorre o mesmo que na disciplina de P2. Essas notas não possui correlação com o tamanho do feedback formativo e com o feedback somativo.

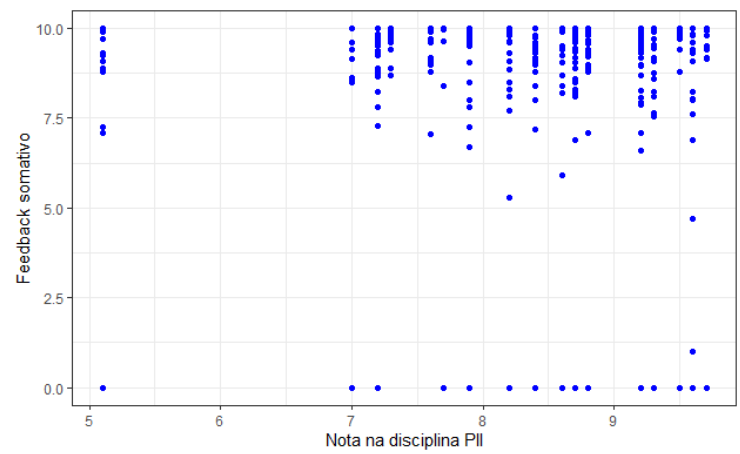

Figura 8. Correlação entre feedback somativo e nota na disciplina P2.

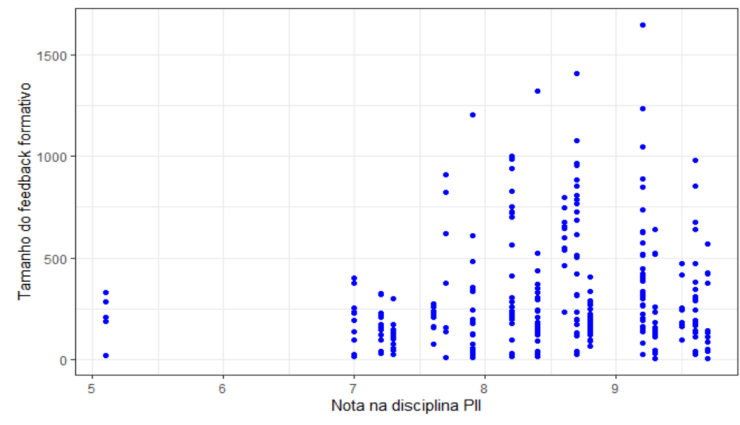

Figura 9. Correlação entre tamanho do feedback formativo e nota na disciplina P2. 


\subsection{Ameaças à validade}

Esse trabalho apresenta algumas ameaças, consideramos o tamanho da amostra um fator limitante, visto foram utilizados dados de apenas um período. Para contornar esse problema, realizamos as análises com os dados acumulados em diferentes laboratórios. Dados provenientes de apenas uma instituição e a falta de aleatoriedade na escolha da amostra, pode ter sido outro fato limitante na nossa pesquisa. Contudo, este estudo demonstrou resultados satisfatórios quanto a análise do papel desempenhado pelo aluno assistente. É importante observar que tais resultados podem ter forte relação com o modelo da assistência, onde o feedback somativo é avaliado com o amparo de uma planilha com itens de verificação.

\subsection{Discussão}

Era esperado que as atividades realizadas por um aluno com mais experiência fosse diferente de um com menos experiência. O estudo realizado neste trabalho mostra que essas atividades podem ser executadas de forma equivalente, independente da experiência especialmente quanto ao feedback somativo gerado. Mas é importante que os alunos sejam instruídos sobre como proceder na atividade, visto que no feedback somativo, o qual é feito de forma guiada, não apresentou diferença em nenhuma das comparações realizadas. Isto é reforçado pela experiência dos professores da disciplina de LP2, que encontram poucos erros das correções realizadas pelos assistentes, bem como poucos pedidos de revisão. O tamanho do feedback formativo apresentou diferença em relação à experiência anterior e no curso apenas quando analisamos os dados acumulados, o que pede por mais análises a serem realizadas, especialmente de natureza qualitativa.

Portanto, vê-se que apesar de vários processos de seleção optarem por candidatos que possuam forte experiência acadêmica prévia, o presente estudo torna possível verificar que muitas vezes esta experiência não é fundamental para a execução das atividades elencadas, ao contário do que se imagina. Dessa maneira, abre um escopo de possibilidades para alunos que, embora capacitados, não possuam uma forte experiência prévia, possibilitando oportunidades para uma parcela maior de atuação ou foco em aspectos não-técnicos.

\section{Conclusão}

Neste trabalho realizamos um estudo de caso a partir dos dados da experiência e das atividades dos alunos assistentes em uma disciplina de programação OO. Avaliamos, e não encrontramos, diferença no feedback somativo em relação as diferentes experiências e encontramos alguma diferença no tamanho do feedback formativo no caso da experiência prévia do assistente.

É necessário fazer uma avaliação e investigação quanto a qualidade do feedback formativo retornado. Apesar dos resultados encontrados sobre o tamanho do feedback, não pudemos responder se há diferença na qualidade do feedback formativo gerado.

No geral, a experiência dos monitores parece ter pouco impacto na sua capacidade de avaliação somativa. Nosso estudo, apesar de inicial, aponta que é preciso reconsiderar um possível víes quanto a seleção de assistentes que façam uso deste critério como forma primária. 


\section{Referências}

Figuerêdo, J. S. L., Machado, J. G., Lima, S. V., da Silva Cerqueira, C. S., and Pereira, C. P. (2021). A experiência da monitoria de algoritmos e programação em cursos de engenharia na perspectiva dos monitores. In Anais do Simpósio Brasileiro de Educação em Computação, pages 183-192. SBC.

Gomes, A. d. J. (2010). Dificuldades de aprendizagem de programação de computadores: contributos para a sua compreensão e resolução. $\mathrm{PhD}$ thesis.

Hattie, J. and Timperley, H. (2007). The power of feedback. Review of educational research, 77(1):81-112.

Mirza, D., Conrad, P. T., Lloyd, C., Matni, Z., and Gatin, A. (2019). Undergraduate teaching assistants in computer science: a systematic literature review. In Proceedings of the 2019 ACM Conference on International Computing Education Research, pages $31-40$.

Santos, L. and Pinto, J. (2018). Ensino de conteúdos escolares: A avaliação como fator estruturante. O ensino na escola de hoje: teoria, investigação e aplicação, pages 503539.

Silva, L. (2021). Avaliação da efetividade das atividades de monitoria no componente curricular de introdução à programação. In Anais do XXIX Workshop sobre Educação em Computação, pages 258-267. SBC. 